Article

\title{
Asymmetric Directional Multicast for Capillary Machine-to-Machine Using mmWave Communications
}

\author{
Jung-Hyok Kwon and Eui-Jik Kim * \\ Department of Convergence Software, Hallym University, 1 Hallymdaehak-gil, Chuncheon-si, \\ Gangwon-do 24252, Korea; jhkwon@hallym.ac.kr \\ * Correspondence: ejkim32@hallym.ac.kr; Tel.: +82-33-248-2333; Fax: +82-33-242-2524
}

Academic Editor: Yonghui Li

Received: 31 January 2016; Accepted: 5 April 2016; Published: 11 April 2016

\begin{abstract}
The huge demand for high data rate machine-to-machine (M2M) services has led to the use of millimeter Wave (mmWave) band communications with support for a multi-Gbps data rate through the use of directional antennas. However, unnecessary sector switching in multicast transmissions with directional antennas results in a long delay, and consequently a low throughput. We propose asymmetric directional multicast (ADM) for capillary M2M to address this problem in mmWave communications. ADM provides asymmetric sectorization that is optimized for the irregular deployment pattern of mulicast group members. In ADM, an M2M gateway builds up asymmetric sectors with a beamwidth of a different size to cover all multicast group members with the minimum number of directional transmissions. The performance of ADM under various simulation environments is evaluated through a comparison with legacy mmWave multicast. The results of the simulation indicate that ADM achieves a better performance in terms of the transmission sectors, the transmission time, and the aggregate throughput when compared with the legacy multicast method.
\end{abstract}

Keywords: asymmetric sectorization; capillary machine-to-machine; directional multicast; Internet of things; millimeter Wave communications

\section{Introduction}

Capillary machine-to-machine (M2M) refers to networking technologies that provide devices with wireless connectivity in a local area by using various communications standards, including Bluetooth Low Energy (BLE), ZigBee, Wi-Fi, and WiGig [1,2]. M2M can be implemented in various intelligent services, such as home automation, smart healthcare, connected automobiles, and smart manufacturing. Therefore, there is consensus in the industry and academia that capillary M2M is a key enabler to realize the Internet of things (IoT) [3-6]. Traditionally, M2M services have been limited to low data rate applications that use wireless sensor networks (WSNs) [7,8]. However, the scope of their use has progressively extended to higher data rate applications due to the increasing demand for real-time streaming of high-definition television (HDTV), high-speed data transfer, and wireless 3D gaming. This trend has led to the adoption of millimeter Wave (mmWave) band communication technologies like IEEE 802.11ad, ECMA-387, and IEEE 802.15.3c, in M2M services [9-11].

mmWave band communication can support a multi-Gbps data rate with directional antennas, and it can be used for various M2M services, particularly for high data rate indoor services such as ad hoc conferences, smart classrooms, and telecasts $[12,13]$. In these applications, the devices that users have can be divided into multiple service groups, and each group might request different multimedia contents such as video streaming, presentation material from a dedicated local server (e.g., projector, presenter's laptop). The local server should support a sufficient data rate to multiple groups 
via a 'group-to-project connection', for which mmWave multicast transmission is an indispensable technology for creation of the seamless interaction service [14-16]. In the mmWave band, the use of directional antennas is considered essential even for multicast transmission due to the short propagation distance problem caused by the unique characteristics of mmWave frequency band such as its oxygen absorption and high path loss [17-19]. Directional antennas form a narrow fan-shape beam in a particular direction, and thus the use of these makes it difficult to cover the entire multicast group consisting of members that are randomly placed around the sender. For multicast transmission with directional antennas, switching the beam direction with a fixed beamwidth in a clockwise or anticlockwise manner has been generally considered [20]. However, this approach may cause excessive power consumption and a long delay. Therefore, the appropriate tuning of the beamwidth and beam direction for multicast transmission is a challenging issue for mmWave band communication [21,22].

Several studies have been carried out to efficiently transmit multicast data with directional antennas. Guo et al. [23] proposed multicast transmission with multiple beam antennas. This method simultaneously covers multicast devices that are scattered around the sender, but it suffers from the high complexity of beamforming and the high cost of installing multiple beam antennas. A one-hop relay transmission for multicast transmission with a directional antenna was proposed in [24,25]. This method effectively extends the transmission range without adjusting the beamwidth. However, the overall network throughput is degraded due to the high overhead of the relay transmission. Sen et al. [26] proposed a priority-based multicast transmission named 'beamcast' in which a sender first transmits packets to nearby multicast devices by using omnidirectional antennas and then transmits packets to the rest of multicast devices using directional antennas. However, this approach exhibits a high performance only when multicast devices are near the sender while unnecessary sectors and duplicate packet reception may also occur.

In this paper, we propose an asymmetric directional multicast transmission (ADM) for a capillary M2M system that uses the minimum number of sectors with different beamwidth sizes to cover multicast devices deployed in a given service area. The operation of the ADM consists of three main phases: Device discovery, Coverage region (CR) allocation, and Asymmetric sectorization. In the device discovery phase, the M2M gateway (GW) discovers multicast group members by using the beamwidth with the smallest size, and it then obtains their sector IDs and locations. Next, during the CR allocation phase, the M2M GW allocates a CR value to each sector by considering the maximum distance of the multicast group member. The $C R$ is a partitioned region within a given sector coverage in which the boundaries have been pre-determined based on a set of transmission ranges of M2M GW. The CR value of the initial sector is determined as the CR ID of the CR for which the farthest multicast group member is located. During asymmetric sectorization, the M2M GW decides the beamwidth size of the new sectors by referring to the allocated CR, and it announces the updated sector information to its multicast group members. An experimental simulation verifies that the ADM can improve the average system throughput by approximately $31 \%$ and can reduce the average transmission time by approximately $20 \%$ when compared to the legacy mmWave multicast approach.

The rest of this paper is organized as follows. Section 2 provides the system model that includes the directional antenna and the mmWave wireless personal area network (WPAN). The detailed operation of the ADM is described in Section 3. An experimental simulation is conducted in Sections 4 and 5 concludes this paper.

\section{System Model}

\subsection{Directional Antenna}

To ensure backwards compatibility and cost efficiency, we consider a conventional sector antenna array instead of an adaptive antenna array. A sector antenna array consists of a number of fixed beams, and one or more beams are combined to form a sector that corresponds to the transmission coverage of the sender. We assume that the direction and the beamwidth of the sector are determined according 
to the beam forming codebook defined in the IEEE 802.15.3c standard [11]. For simplicity of the planar analysis for the mmWave band communication, we assume that all M2M devices exist in the same plane and use a two-dimensional flat-top directional antenna.

Figure 1 shows an example of the two-dimensional flat-top directional antenna model. This model has a constant antenna gain only within the beamwidth, and the antenna gains of the main lobe $\left(G_{m}\right)$ and the side lobe $\left(G_{S}\right)$ are ' $2 \pi$ /beamwidth' and ' 0 ', respectively. Considering that the beamwidth of each beam is $\theta$, the beamwidth of a sector can be chosen from $\theta$ to $2 \pi$ in $\theta$ units. Therefore, the maximum number of sectors is ' $2 \pi / \theta$ ' with a minimum number of sectors of ' 1 '. Meanwhile, the maximum transmission range of the antenna is represented as $\mathrm{TR}$, which can be calculated using Equation (1).

$$
\mathrm{TR}=10^{\frac{\kappa}{10 n}},\left(\kappa=P_{t}+G_{t}+G_{r}-I_{L}-P L_{0}-\gamma_{t h}\right)
$$

where $P_{t}$ is the transmission power, $G_{t}$ and $G_{r}$ are the transmitter and receiver antenna gains, $I_{L}$ is the implementation loss, $P L_{0}$ is the path loss at $1 \mathrm{~m}, \gamma_{t h}$ is the receiver sensitivity, and $n$ is the path loss exponent. Table 1 lists an example of the maximum transmission range of the directional antennas for varying beamwidths and the modulation and coding schemes (MCSs). The beamwidth size is smaller and the transmission range is longer because the sector with a small beamwidth has a high antenna gain. Moreover, the transmission range varies according to the MCS, which affects the receiver sensitivity of the M2M devices.

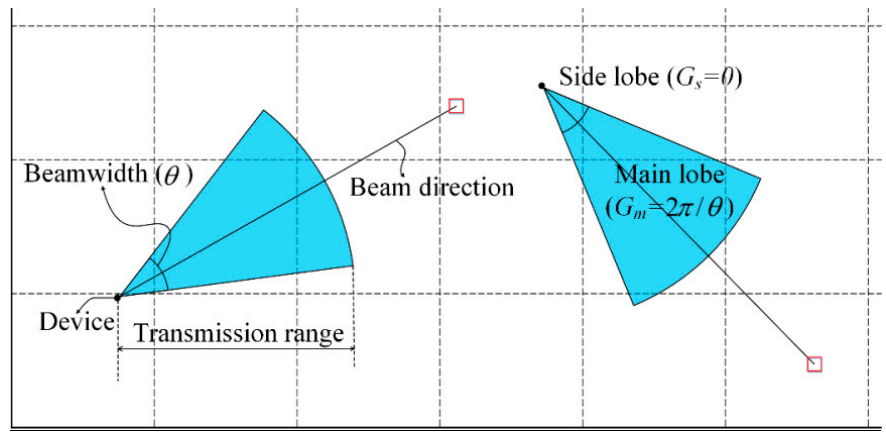

Figure 1. Two-dimensional flat-top directional antenna model.

Table 1. Example of the maximum transmission range of the directional antennas.

\begin{tabular}{lccc}
\hline \multicolumn{1}{c}{ MCS } & Sensitivity (dB) & BeamWidth (rad) & $\begin{array}{c}\text { Maximum } \\
\text { Transmission Range (m) }\end{array}$ \\
\hline Data rate: 412 Mbps & & $\pi / 18$ & 60.47 \\
Modulation: BPSK & -61 & $\pi / 9$ & 30.23 \\
Spreading factor: 4 & $\pi / 6$ & 15.11 \\
FEC type: RS(255,239) & & $\pi / 3$ & 10.07 \\
& & $\pi$ & 3.35 \\
\hline Data rate: 825 Mbps & -58 & $\pi / 18$ & 42.81 \\
Modulation: BPSK & & $\pi / 9$ & 21.40 \\
Spreading factor: 2 & & $\pi / 6$ & 14.27 \\
FEC type: RS(255,239) & & $\pi / 3$ & 7.13 \\
& & $\pi$ & 2.37 \\
\hline Data rate: 1650 Mbps & -55 & $\pi / 18$ & 30.30 \\
Modulation: BPSK & & $\pi / 9$ & 15.15 \\
Spreading factor: 1 & $\pi / 6$ & 10.10 \\
FEC type: RS(255,239) & $\pi / 3$ & 5.05 \\
& & $\pi$ & 1.68 \\
\hline
\end{tabular}




\subsection{Network Model}

We consider a centralized network model where a number of M2M devices form a capillary $\mathrm{M} 2 \mathrm{M}$ and one of those becomes an M2M GW that acts as a personal area network coordinator (PNC). The M2M GW performs various procedures including neighbor discovery, synchronization, channel time scheduling, and network termination to manage the overall capillary M2M network. For multicast transmission with a directional antenna, the M2M GW manages the multicast group members, announces multicast information and assigns the channel time. Moreover, it transmits multicast packets by switching the direction of the antennas. Considering that all M2M devices use a directional antenna, M2M GW should select a proper beamwidth size for the sector and should schedule a sector switching timing to cover all sectors.

In this paper, we consider the single carrier mode of mmWave physical layer (SC-PHY) specified in the IEEE 802.15.3c WPAN standard [11,27]. SC-PHY is designed to support the low complexity and the high energy-efficiency, and specifies three classes for 14 MCSs, which are listed in Table 2. Thus, according to their supported data rates, the diverse mmWave applications can be served. The SC-PHY frame includes the PHY preamble, the frame header, and the payload. The PHY preamble prior to the frame header supports frame detection, frame synchronization, and channel estimation. The frame header includes PHY header, medium access control (MAC) header, and header check sequence (HCS) for the frame decoding. Finally, the payload includes the data, and its maximum length is supported up to 1 Mbytes.

Table 2. MCS categorization of mmWave SC-PHY.

\begin{tabular}{ccc}
\hline MCS Class & MCS ID & Data Rate (Mbps) \\
\hline & 0 & 25.8 \\
& 1 & 412 \\
Class 1 & 2 & 825 \\
& 3 & 1650 \\
& 4 & 1320 \\
& 5 & 440 \\
6 & 880 \\
\hline \multirow{2}{*}{ Class 2 } & 7 & 1760 \\
& 8 & 2640 \\
& 9 & 3080 \\
& 10 & 3290 \\
Class 3 & 11 & 3300 \\
& 12 & 3960 \\
\hline
\end{tabular}

\section{Design of the ADM}

In the transmission with directional antennas, the M2M GW should repeat the transmission for the same packet as the number of formed sectors in order to cover all multicast group members. This causes the degradation of the network performance in terms of the throughput and delay. Therefore, it is necessary to minimize the redundant multicast transmissions by reducing the number of sectors within the capillary M2M network. The ADM is designed to build up an optimal number of asymmetric sectors for directional multicast transmissions by taking the distribution of M2M devices into account. Note that 'asymmetric' means that each sector could maintain a beamwidth of a different size with the corresponding difference in the transmission range. In this section, we describe the design of the ADM in detail. As mentioned earlier, the operation of the ADM consists of three phases: (1) Device discovery, (2) CR allocation, and (3) Asymmetric sectorization.

During device discovery, the capillary M2M network operates over the initialized 'symmetric' sectors that have a unit beamwidth. Hereafter, this is referred to as the unit-beam sector. The M2M 
GW broadcasts multicast information via a beacon for all unit-beam sectors, and each multicast group member responds with a join request message. From the join requests, the M2M GW obtains the information for multicast group members, including the device ID, current sector ID, and distance to the M2M GW. The distance to each M2M device from the M2M GW can be represented using the matrix $\mathbf{D}_{(i)}$, as in Equation (2).

$$
\mathbf{D}_{(i)}=\left[d_{(i, 1)}, d_{(i, 2)}, \cdots, d_{(i, j)}, \cdots, d_{\left(i, N_{i}\right)}\right], 0<i \leqslant M, 0<j \leqslant N_{i}
$$

where $i$ is a unit-beam sector ID, $j$ is an M2M device ID within the unit-beam sector $i, M$ is the total number of unit-beam sectors, $N_{i}$ is the number of M2M devices within the unit-beam sector $i$, and $d_{(i, j)}$ is the distance value between the M2M GW and device $j$ within the unit-beam sector $i$.

During the CR allocation phase, the M2M GW allocates a CR value to each unit-beam sector. Figure 2 describes the structure of the CR. In the figure, the unit-beam sector is virtually divided into multiple CRs with a unique CR ID, $k$, and their boundaries are defined as the transmission ranges for varying beamwidths (refer to Table 1.). In other words, the CR is a partitioned region within the coverage of a unit-beam sector, and its area is pre-determined based on a set of transmission ranges of the M2M GW, which is represented by the matrix $\mathbf{R}$, as in Equation (3).

$$
\mathbf{R}=\left[r_{(\theta)}, r_{(2 \theta)}, \cdots, r_{(k \theta)}, \cdots, r_{(2 \pi)}\right], 0<k \leqslant 2 \pi \theta^{-1}
$$

where $\theta$ is a beamwidth of unit-beam sector, $k$ is a CR ID, and $r_{(k \theta)}$ is a CR boundary of $\mathrm{CR}_{k}$, which is the same as the transmission range of the M2M GW when the beamwidth size is $k \theta$.
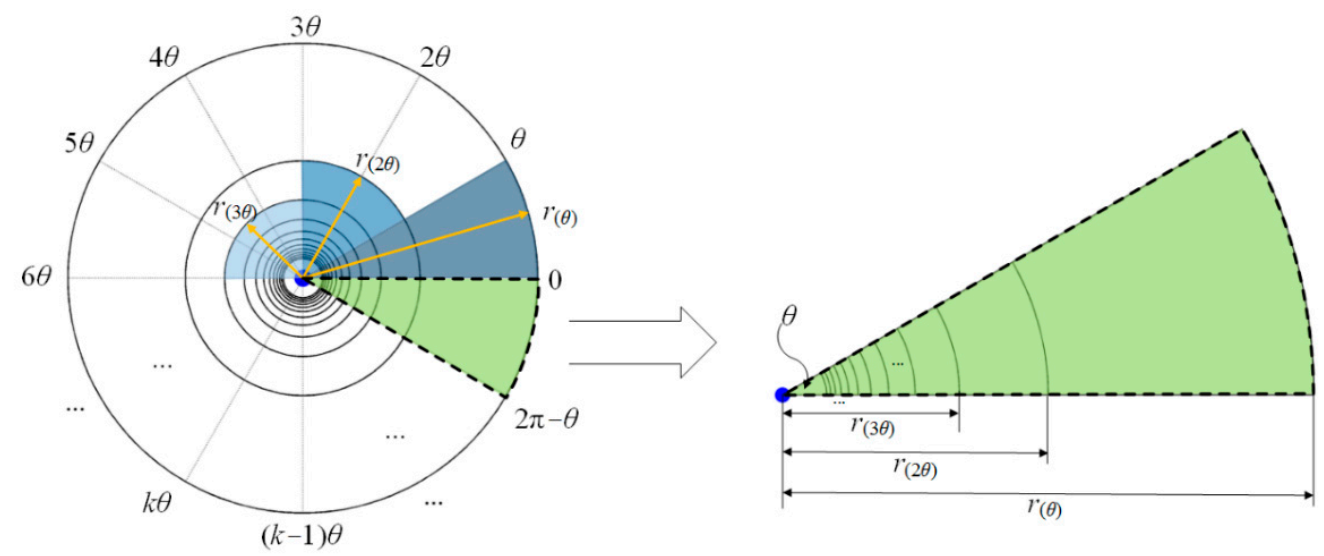

Figure 2. Coverage region.

The CR value of unit-beam sector is determined as the CR ID of CR where the farthest M2M device is located. Specifically, to allocate a CR value, the M2M GW searches the maximum distance, $\max \left(d_{(i, j)}\right)$ within the matrix $\mathbf{D}_{(i)}$, and then the CR value of the unit-beam sector $i, c r(i)$ is determined as $k$ (i.e., the CR ID of CR containing the device with $\left.\max \left(d_{(i, j)}\right)\right)$, as follows.

$$
c r_{(i)}=\left\{\begin{array}{l}
k, r_{((k+1) \theta)} \leqslant \max \left(d_{(i, j)}\right)<r_{(k \theta)} \\
0, \mathbf{D}_{(i)}=\phi
\end{array}\right.
$$

Note that, if there is no device within a unit-beam sector, the M2M GW sets the CR value to zero. The CR value of the each unit-beam sector is represented by the matrix $\mathbf{C R}$, as in Equation (5).

$$
\mathbf{C R}=\left[c r_{(1)}, c r_{(2)}, \cdots, c r_{(i)}, \cdots, c r_{(M)}\right], 0<i \leqslant M
$$


In the asymmetric sectorization phase, the M2M GW conducts an asymmetric sectorization procedure where the sectors of the directional antenna are newly configured, and each sector (which is hereafter referred to as an asymmetric sector) can maintain different beamwidth size and obtain a newly allocated unique sector ID. The result of the asymmetric sectorization procedure is represented as the matrix AS, as in Equation (6).

$$
\mathbf{A S}=\left[a s_{(1)}, a s_{(2)}, \cdots, a s_{(i)}, \cdots, a s_{(M)}\right], 0<i \leqslant M
$$

where $a s_{(i)}$ is an asymmetric sector ID, $i$ is a unit-beam sector ID, and $M$ is the total number of unit-beam sectors. In Equation (6), the same multiple values of $a s_{(i)}$ stand for one asymmetric sector, and Algorithm 1 describes the asymmetric sectorization procedure. In this algorithm, M2M GW repeatedly conducts the 'sector decision', thereby deciding whether the adjacent unit-beam sectors can be merged or not. M2M GW initializes the variables (i.e., init_pt, decision_index, numMergeSector, empty_cnt, numFrontSector, and numRearSector) that are needed to configure the asymmetric sectors. 'init_pt' denotes the starting point for each sector decision, which is an ID of the foremost unit-beam sector, and it is updated whenever the sector decision is newly performed. 'decision_index' indicates the number of times that the sector decision has been performed, and 'numMergeSector' is the number of unit-beam sectors that can be merged at each sector decision. 'empty_cnt' is the number of unit-beam sectors with no device (i.e., empty sector). Thus the difference of 'decision_index' and 'empty_cnt' indicates the number of asymmetric sectors. Finally, 'numFrontSector' and 'numRearSector' indicate the numbers of unit-beam sectors with the same $a s_{(i)}$ from the starting point $(i . e ., i=1)$ and from the end point (i.e., $i=M)$, respectively.

The sector decision is iterated for each unit-beam sector to obtain the asymmetric sector ID, $a s_{(i)}$, that has been allocated. First, the empty sector is allocated with $a s_{(i)}=0$. For the unit-beam sectors with devices, M2M GW compares the CR value of each unit-beam sector with numMergeSector. At the beginning of the asymmetric sectorization phase, numMergeSector is initialized to 1 , and it is used as a reference value for the merging of adjacent unit-beam sectors. As a result of each sector decision, M2M GW can merge adjacent unit-beam sectors into one transmission sector referring the number of numMergeSector. If all CR values are equal to or larger than numMergeSector for the unit-beam sectors from init_pt to $i$, the same $a s_{(i)}$ is allocated to the corresponding unit-beam sectors. Note that $a s_{(i)}$ is obtained as (decision_index - empty_cnt). The SectMergeFlag is used to verify the result of the comparison between the $\mathrm{CR}$ value and numMergeSector. Therefore, in the case where the $\mathrm{CR}$ values for every checked unit-beam sector are equal to or larger than numMergeSector, SectMergeFlag is set to TRUE and otherwise to FALSE. When all unit-beam sectors are allocated $a s_{(i)}, \mathrm{M} 2 \mathrm{M} \mathrm{GW}$ finally conducts the sector decision for the front and the rear unit-beam sectors to check to merge them. If all CR values for the front and the rear unit-beam sectors are equal to or larger than the sum of numFrontSector and numRearSector, $a_{(i)}$ for the rear unit-beam sectors is updated to 1 . The unit-beam sectors with the same asymmetric sector ID are merged into one asymmetric sector, and as a result of this algorithm, M2M GW can build up the optimal sectorization for multicast transmission.

Figure 3 shows an operational example of the asymmetric sectorization. In the figure, M2M GW is assumed to use the unit-beam sector with a beamwidth of $\pi / 4$, which is rotated in an anti-clockwise direction. We also assume that the matrix $\mathbf{C R}$ is given by $[5,8,4,4,0,2,3,1]$. At the 1 st unit-beam sector, M2M GW checks $c r_{(1)}=5$ to see whether it is larger than numMergeSector (1). If so, it allocates 1 to $a s_{(1)}$ and increases numMergeSector by 1 . Then, at the 2nd unit-beam sector, $c r_{(1)}$ and $c r_{(2)}$ are compared with the updated numMergeSector (2). If both are larger than 2, it also allocates 1 to $a s_{(2)}$. This procedure is continued until $\mathrm{Cr}_{(i)}$ is smaller than numMergeSector. Accordingly, at the 3rd and 4th unit-beam sectors, both $a s_{(3)}$ and $a s_{(4)}$ are allocated to $1_{\text {. as }} a_{(5)}$ is allocated to 0 (decision_index is increased by 1 and numMergeSector is initialized to 1 ) because the 5 th unit-beam sector is the empty sector (i.e., $c r_{(5)}=0$ ). $a_{(6)}$ and $a s_{(7)}$ are allocated to 2 considering the empty sector $\left(c r_{(5)}=0\right)$. Likewise, $a s_{(8)}$ of the last unit-beam sector gets allocated 3. Finally, M2M GW checks whether the front and the rear sectors can 
be merged. In our example, this is impossible because $c r_{(3)}=4, c r_{(4)}=4$, and $c r_{(8)}=1$ are smaller than 5 (numFrontSector + numRearSector). Consequently, M2M GW newly builds up three asymmetric sectors, and their beamwidth sizes are $\pi, \pi / 2$, and $\pi / 4$, respectively.

Algorithm 1 Asymmetric sectorization

1. INITIALIZE init_pt to 1, decision_index to 1, numMergeSector to 1, empty_cnt to 0 , SectMergeFlag to TRUE / / variables are initialized

2. FOR each unit-beam sector, $i, i \in[1, M] / / M$ is the number of unit-beam sectors

3. $\quad$ IF $c r_{(i)}==0 / /$ unit-beam sector without device

4. $\quad a_{(i)} \leftarrow 0 / /$ assign zero for asymmetric sector ID

5. decision_index $\leftarrow$ decision_index $+1 / /$ change temporary sector index

6. empty_cnt $\leftarrow$ empty_cnt +1

7. $\quad$ init_pt $\leftarrow i+1$

8. $\quad C R \_$index $\leftarrow 0$

9. $\quad$ ELSE / / unit-beam sector with device

10. FOR each unit-beam sector, $j, j \in[$ init_pt, $i]$

11. IF $c r_{(i)} \geqslant$ numMergeSector // compare each CR value with numMergeSector

12. $\quad$ SectMergeFlag $\leftarrow$ TRUE

13. ELSE

14. SectMergeFlag $\leftarrow$ FALSE

$15 . \quad$ break

16. ENDIF

17. ENDFOR

18. IF SectMergeFlag $==$ TRUE

19. $\quad$ numMergeSector $\leftarrow$ numMergeSector +1

20. ELSE

21. decision_index $\leftarrow$ decision_index $+1 / /$ change decision index

22. $\quad$ init_pt $\leftarrow i$

23. numMergeSector $\leftarrow 2$

24. ENDIF

25. $\quad a_{(i)} \leftarrow$ decision_index - empty_cnt / / assign value for asymmetric sector ID

26. ENDIF

27. IF $i==M_{\text {a }} \& a s_{(i)} !=0 / /$ current unit-beam sector is the last one

28. numFrontSector $\leftarrow$ number of unit-beam sectors where $a s_{(i)}$ is 1

29. numRearSector $\leftarrow$ number of unit-beam sectors where $a_{(i)}$ is (decision_index - empty_cnt)

30. $\quad$ IF $\mathrm{Cr}_{(i)} \geqslant$ (numFrontSector + numRearSector)

31. $\forall a s_{(k)} \leftarrow 1, k \in[$ init_pt, $M] / /$ merge the last sector

32. ENDIF

33. ENDIF

34. ENDFOR 


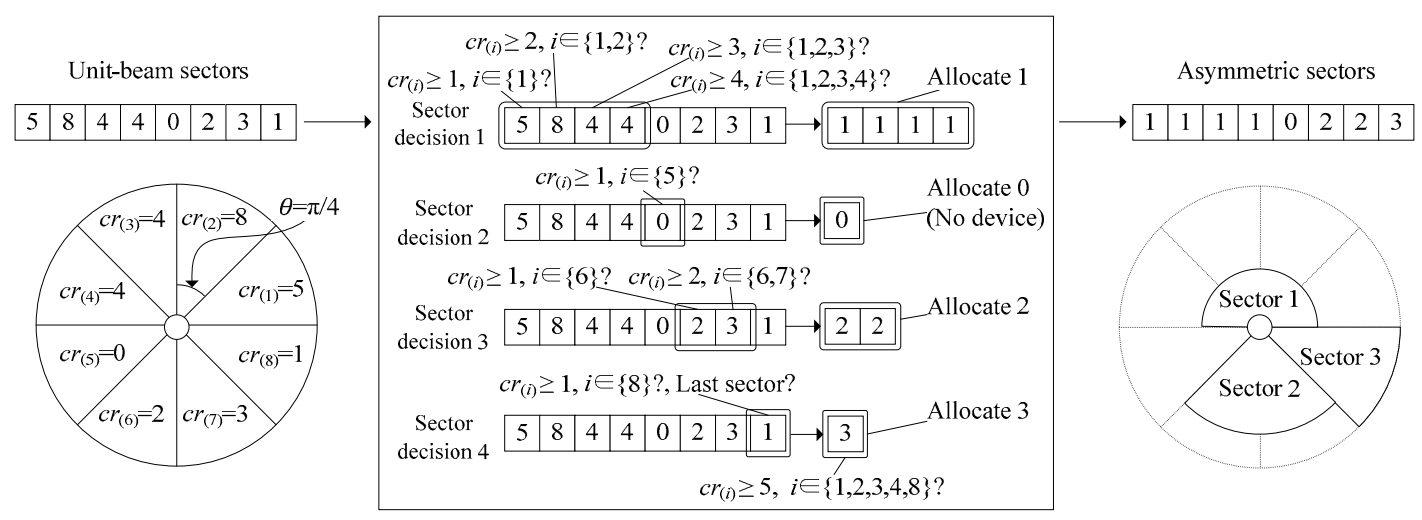

Figure 3. Example of asymmetric sectorization.

\section{Performance Evaluation}

We evaluate the performance of the ADM by conducting an experimental simulation. Various performance metrics are considered in this simulation in terms of the number of transmission sectors, transmission time, and aggregate throughput. We compare the results of the simulation to the multicast transmission approach of the IEEE 802.15.3c standard in order to verify the superiority of ADM. In the following subsections, we describe the simulation setting and configuration and discuss the results of the simulation, in detail.

\subsection{Simulation Setting and Configuration}

In the simulation, we consider a conference room scenario, where it is assumed that a number of M2M devices are randomly deployed within the $\mathrm{W} \times \mathrm{L}$ size room and divided into three multicast groups (MGs) (i.e., MG1, MG2, and MG3). The MGs are supposed to be served sequentially from the M2M GW at the center of the room. To support 1080p HD video streaming, we use $1.65 \mathrm{Gbps}$ data rate and a $29.63 \mu$ s transmission interval since it requires more than $1.485 \mathrm{Gbps}$ data rate and less than $29.64 \mu$ s transmission interval [28]. Considering an HD video streaming application for three MGs, we configure packet of different sizes for each MG, which represents the chunk of data for different video streaming content [29-32]. We assume that the ADM is implemented on top of the IEEE 802.15.3c PHY layer model and its operational phases are independent with the superframe structure of IEEE 802.15.3c MAC layer. For fair comparison of the network performance, both in ADM and legacy multicast, it is assumed that the capillary M2M network consists of stationary devices, thus the operation of device discovery is performed only once at the time of deployment throughout the whole network lifetime using the unit-beam sectors. However, considering the information types of control messages, we further assume that ADM and legacy multicast use control messages of 50 byte- and 20 byte-size, respectively. The simulation is iterated 50 times for different room sizes, transmission ranges, and beamwidth sizes of the unit-beam sector. The detailed parameters are listed in Table 3.

Table 3. Simulation parameters.

\begin{tabular}{cccc}
\hline Parameter & Value & Parameter & Value \\
\hline PHY & IEEE $802.15 .3 \mathrm{c}$ & Antenna & Sectored antenna \\
Traffic application & CBR & Slot time & $20 \mu \mathrm{s}$ \\
Beamwidth of unit-beam sector & $\pi / 18, \pi / 9, \pi / 6$ & SIFS & $2.5 \mu \mathrm{s}$ \\
MG1 packet size & $2 \mathrm{Kbytes}$ & Preamble duration & $8.157 \mu \mathrm{s}$ \\
MG2 packet size & $3 \mathrm{Kbytes}$ & Transmission power & $20 \mathrm{~mW}$ \\
MG3 packet size & 4 Kbytes & Receive power & $15 \mathrm{~mW}$ \\
Data rate & $1.65 \mathrm{Gbps}$ & Idle power & $10 \mathrm{~mW}$ \\
Sensitivity & $-55 \mathrm{dBm}$ & Guard time & $0.02 \mu \mathrm{s}$ \\
\hline
\end{tabular}




\subsection{Simulation Results}

Figure 4 shows the number of transmission sectors for various unit-beam sectors. The number of transmission sectors is the same as repeated directional transmissions for one multicast, and thus it works as system overhead. In the simulation, we use three unit-beam sectors with different beamwidth sizes set to $\pi / 18, \pi / 9$, and $\pi / 6$, respectively. The devices are randomly deployed within a transmission range of M2M GW, and the number of devices varies from 10 to 100. In ADM, the number of sectors slowly increases as the number of devices increases. ADM tries to maintain the minimum number of sectors by merging the adjacent unit-beam sectors in accordance with the location of the devices. On the other hand, for the legacy multicast, this sharply increases until reaching the number of unit-beam sectors of the initial simulation settings. Specifically, for one multicast transmission, ADM maintains $34.84 \%, 23.59 \%$, and $19.06 \%$ fewer sectors than legacy multicast on average, when the beamwidths of unit-beam sector are $\pi / 18, \pi / 9$, and $\pi / 6$, respectively.

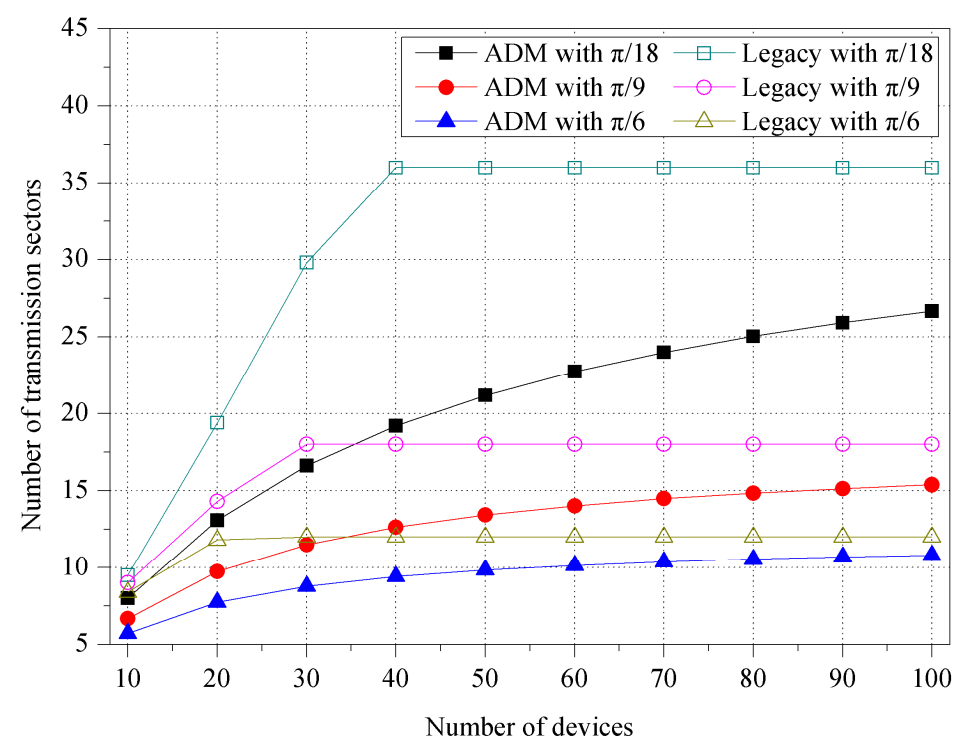

Figure 4. Number of transmission sectors for various unit-beam sectors.

Figure 5 shows the impact of the room size. We assume three square-shaped conference room environments with different areas of $24 \times 24 \mathrm{~m}^{2}, 12 \times 12 \mathrm{~m}^{2}$, and $8 \times 8 \mathrm{~m}^{2}$, respectively. The M2M GW is placed in the center of the room, and it uses the unit-beam sector with $\pi / 18$ beamwidth. In the room with a size of $8 \times 8 \mathrm{~m}^{2}$, the ADM exhibits the minimum number of sectors since it can use the asymmetric sectors with the widest bandwidth to cover the room. Specifically, the average number of sectors for ADM is 19.23 (in $24 \times 24 \mathrm{~m}^{2}$ ), 12.23 (in $12 \times 12 \mathrm{~m}^{2}$ ), and 8.93 (in $8 \times 8 \mathrm{~m}^{2}$ ), respectively. However, the legacy multicast maintains almost the same number of sectors regardless of the room size, and in each room environment, the ADM exhibits approximately $38.10 \%, 60.97 \%$, and $71.30 \%$ less number of sectors compared to the legacy multicast.

Figure 6 shows the transmission time for various beamwidth sizes of the unit-beam sector. The number of packets per a multicast group in the x-axis means the number of chunks that are continuously transmitted in one transmission. As in Figure 5, the unit-beam sector of the M2M GW has beamwidths of $\pi / 18, \pi / 9$, and $\pi / 6$, respectively, to provide an appropriate transmission range according to each room size (i.e., $24 \times 24 \mathrm{~m}^{2}, 12 \times 12 \mathrm{~m}^{2}$, and $8 \times 8 \mathrm{~m}^{2}$ ). In the figure, the use of a small beamwidth increases the transmission time due to more sector switchings (i.e., a larger number of transmissions) to complete one multicast. Specifically, when the number of packets is 6 , the transmission time for ADM decreases from $5.23 \mathrm{~ms}$ (beamwidth of $\pi / 18$ ) to $2.66 \mathrm{~ms}$ (beamwidth of $\pi / 6$ ). When compared to legacy multicast, ADM exhibits an approximately $31 \%$ better performance in terms of the transmission time. 


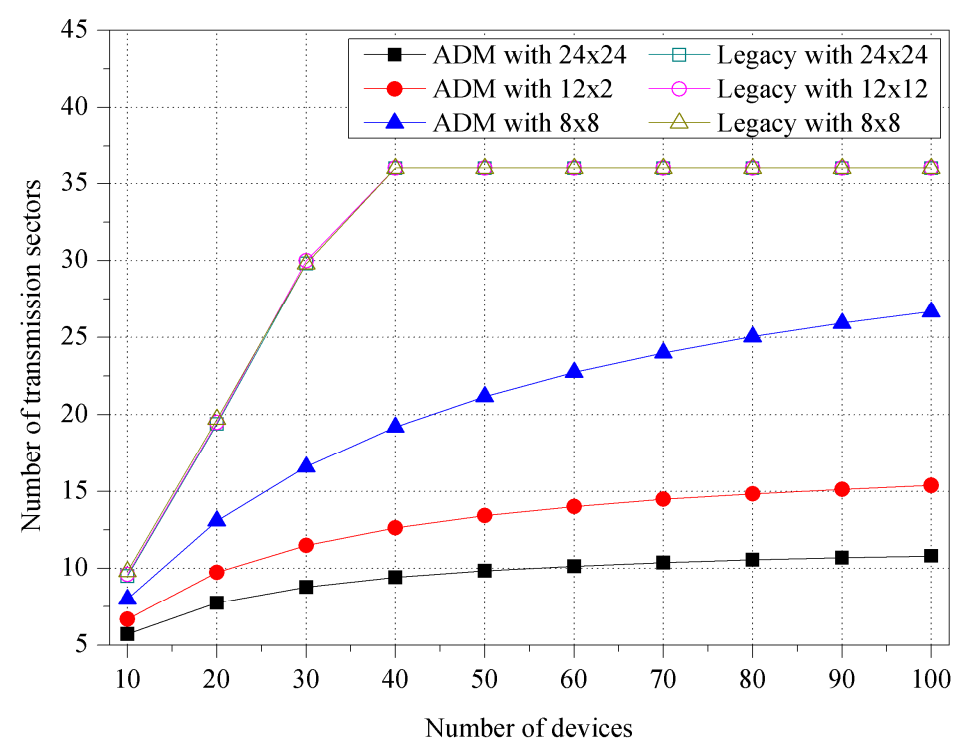

Figure 5. Number of transmission sectors for various room sizes.

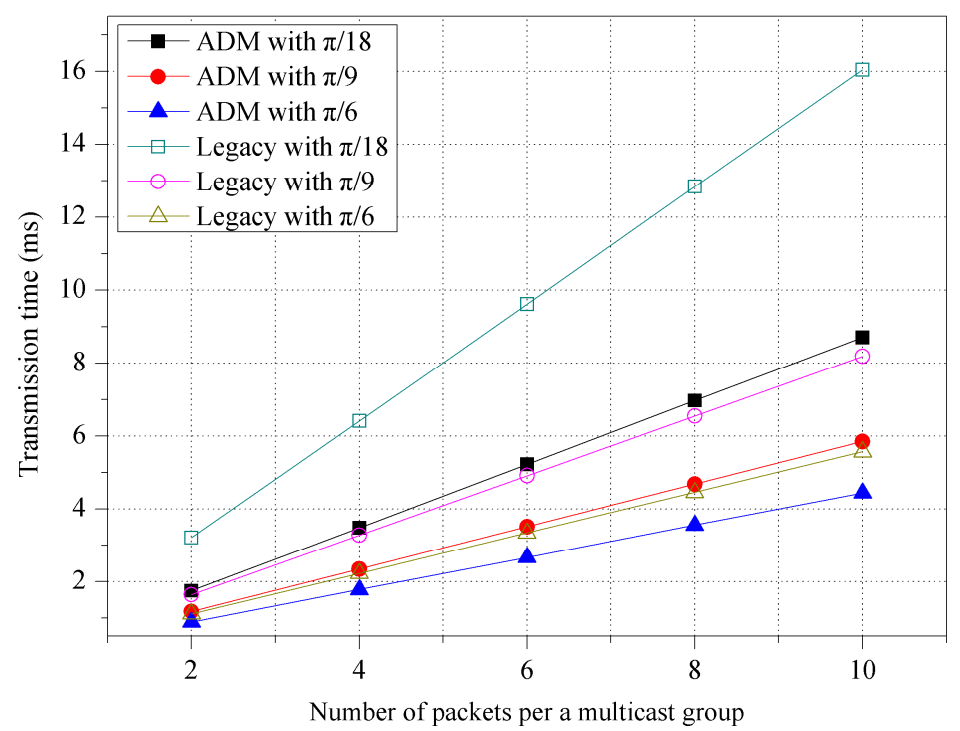

Figure 6. Transmission time for various unit-beam sectors.

Figure 7 shows the transmission time for various room shapes. The network performance of $\mathrm{ADM}$ is affected by the shape of the room while that of legacy multicast is not. In a rectangular room (i.e., $9 \times 16 \mathrm{~m}^{2}$ ), ADM exhibits a transmission time that is $11.31 \%$ smaller than that in a square room (i.e., $12 \times 12 \mathrm{~m}^{2}$ ). The devices in the rectangular room tend to be placed near the M2M GW, and ADM is thus likely to maintain a smaller number of sectors with a large beamwidth. On the other hand, for legacy multicast, M2M GW has to maintain the same number of sectors regardless of the room shape. The results of the simulation show that ADM obtains a transmission time that is $32.30 \%$ smaller than that of legacy multicast in a rectangular room. Considering that most conference rooms are rectangular, ADM could therefore be useful for real environments. 


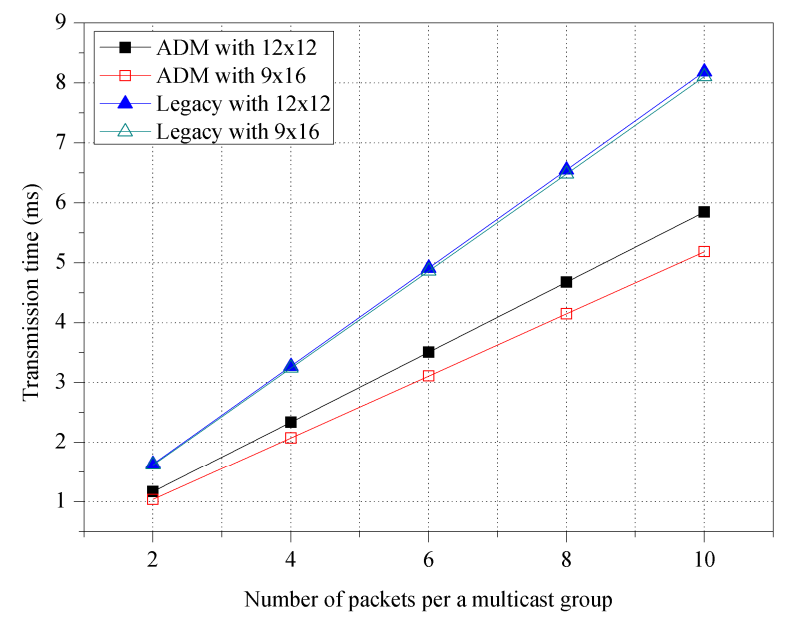

Figure 7. Transmission time for various room shapes.

We evaluate the aggregate throughput, which can be estimated as follows [33].

$$
S=\frac{E[P] \sum_{i=1}^{N} n_{i}}{\sum_{i=1}^{N} E\left[T_{i}\right]}
$$

where $N$ is the number of sectors, $n_{i}$ is the number of devices within the sector $i, E[P]$ is an average length of the packet payload, and $E\left[T_{i}\right]$ is the average transmission time at sector $i$. Regarding the evaluation of the aggregate throughput, we consider various experimental factors, such as the beamwidth size of the unit-beam sector, the shape of room, and the position of M2M GW.

First of all, Figure 8 shows the impact of the beamwidth size of unit-beam sector. In both $\mathrm{ADM}$ and legacy multicast, the aggregate throughput increases as the beamwidth size of unit-beam sector increases due to the reduced switching delay of the directional transmission. With asymmetric sectorization under the same environment, ADM achieves approximately $19.91 \%$ higher aggregate throughput compared with legacy multicast.

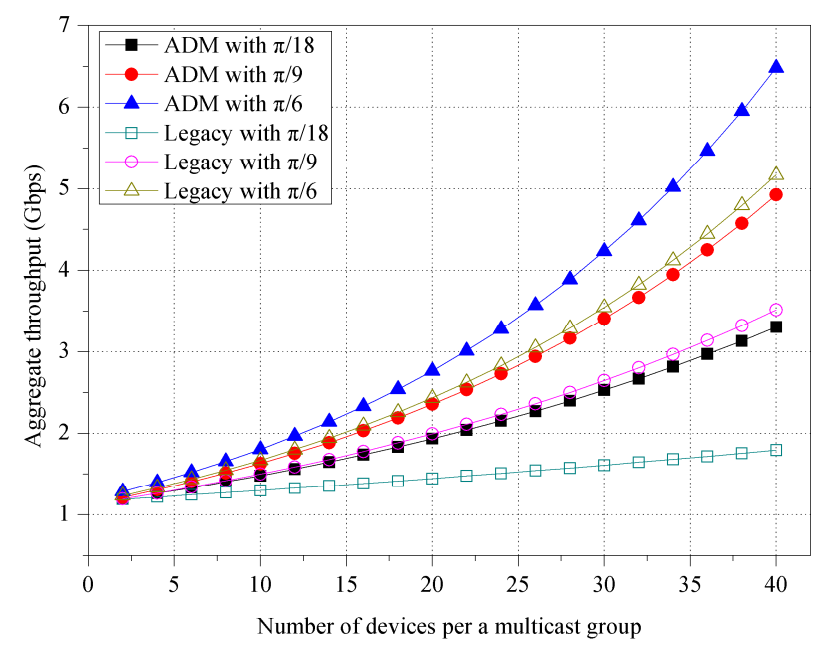

Figure 8. Aggregate throughput for various unit-beam sectors.

Figure 9 shows the impact of the room shape on the aggregate throughput. In the simulation, the beamwidth of the unit-beam sector is set to $\pi / 9$. As seen in Figure 7 , ADM exhibits a shorter 
transmission time in a room with a rectangular shape and likewise obtains an improved throughput performance. In the figure, ADM exhibits $7.40 \%$ higher throughput on average when compared to the case of a square room. However, the throughput of legacy multicast is not affected by the room shape.

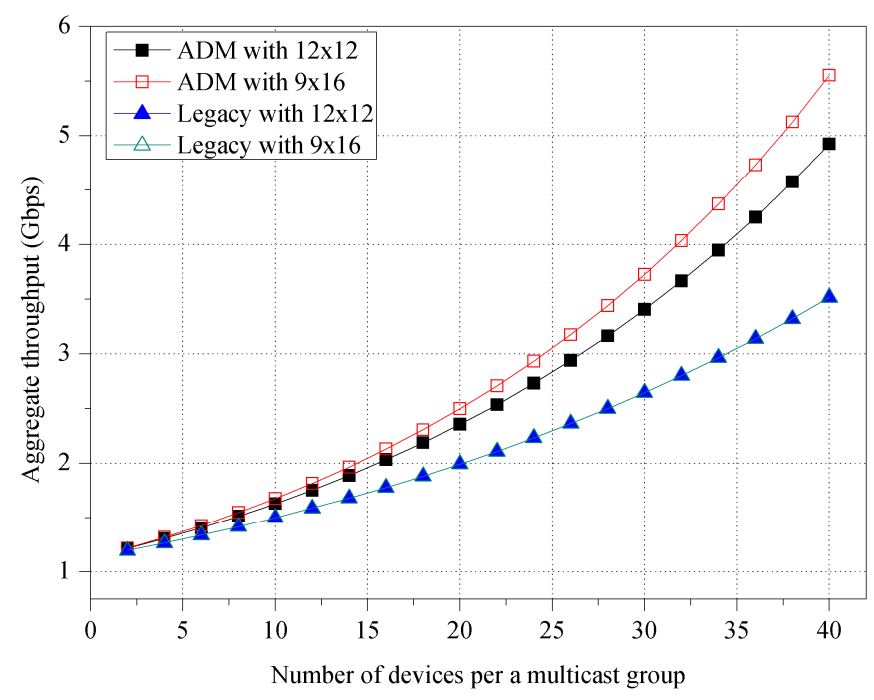

Figure 9. Aggregate throughput for various room shapes.

As mentioned earlier, the sectorization of ADM is based on the distance of each device from the M2M GW, and it is thus necessary to evaluate the impact of the position of the M2M GW in the conference room. In Figure 10, we measure the aggregate throughput for various positions of M2M GW. M2M GW is initially placed in the center (i.e., $\mathrm{GW}(0,0))$ of a $12 \times 12 \mathrm{~m}^{2}$ room, and it then moves upward by $3 \mathrm{~m}$ (i.e., $\mathrm{GW}(0,3)$ ) and $6 \mathrm{~m}$ (i.e., $\mathrm{GW}(0,6))$. The beamwidth of the unit-beam sector is set to $\pi / 9$. In both the ADM and legacy multicast, the aggregate throughput increases as the M2M GW moves from the center. When M2M GW is located at the biased position in the room, the number of empty sectors increases, and the other sectors are likely to include more devices. In the simulation, we configure the M2M GW to not transmit any packet at the empty sector with no device. Thus, they commonly exhibit higher throughput performance in the biased position. Specifically, the ADM shows a throughput that is higher by $11.53 \%$ on average when compared with legacy multicast as a result of the difference in the number of sectors.

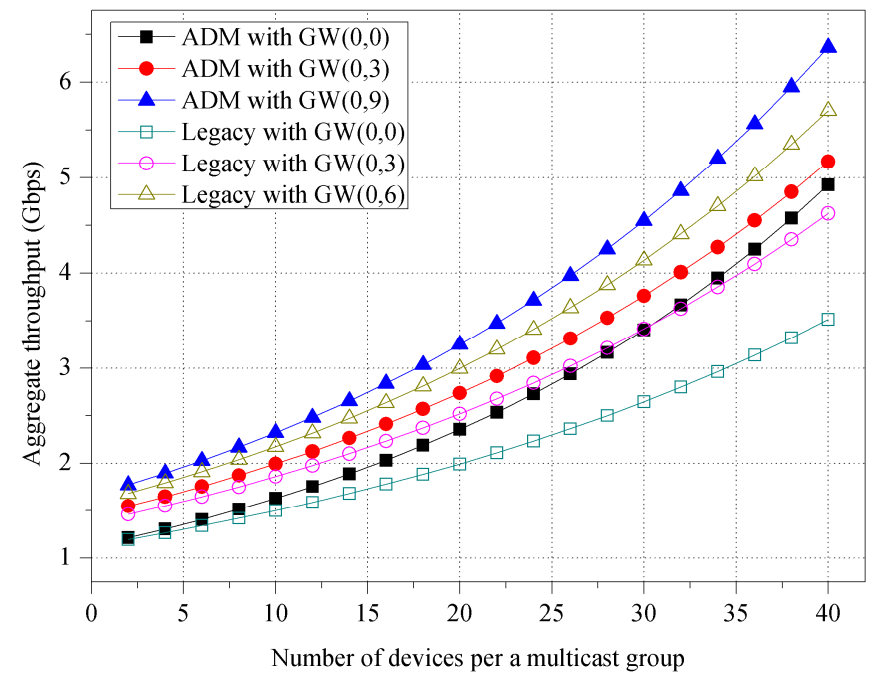

Figure 10. Aggregate throughput for various positions of M2M GW. 
We investigate the control overhead of ADM. The operation of the device discovery incurs the control overhead due to control messages (i.e., join-request and response messages) that are exchanged in a carrier sense multiple access with collision avoidance (CSMA/CA) manner. This overhead problem is common for both ADM and legacy multicast. This is because the same unit-beam sector is used in the device discovery phase, thus the same numbers of control messages are required. However, each mechanism uses a control message of a different size (i.e., ADM: 50 bytes, legacy multicast: 20 bytes), thereby mainly causing the difference of control overhead between ADM and legacy multicast.

Figures 11 and 12 show the control overhead for the control message exchange with respect to the energy consumption per a device and the transmission time, respectively. In the figures, we consider the overhead only for the transmission and reception of the control messages. The ADM exhibits $6.79 \%$ higher energy consumption and $5.43 \%$ longer transmission time compared to the legacy multicast. Table 4 shows the control overhead of the entire device discovery phase including the channel access operation of CSMA/CA, where the ADM exhibits much smaller difference of control overhead compared to the previous results. Specifically, ADM exhibits $0.89 \%$ higher energy consumption and $0.78 \%$ longer transmission time. This is because the impact of difference on the control message sizes is reduced due to the CSMA/CA backoff delay. This overhead problem can be ignored over a long period of network operation when considering that the device discovery is performed only once at the time of deployment throughout the whole network lifetime.

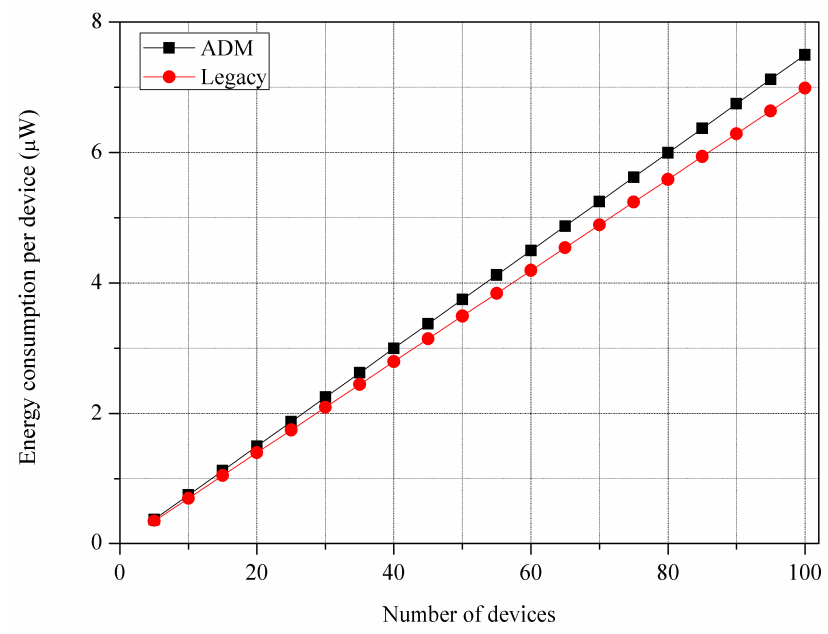

Figure 11. Energy consumption per device for control message exchange.

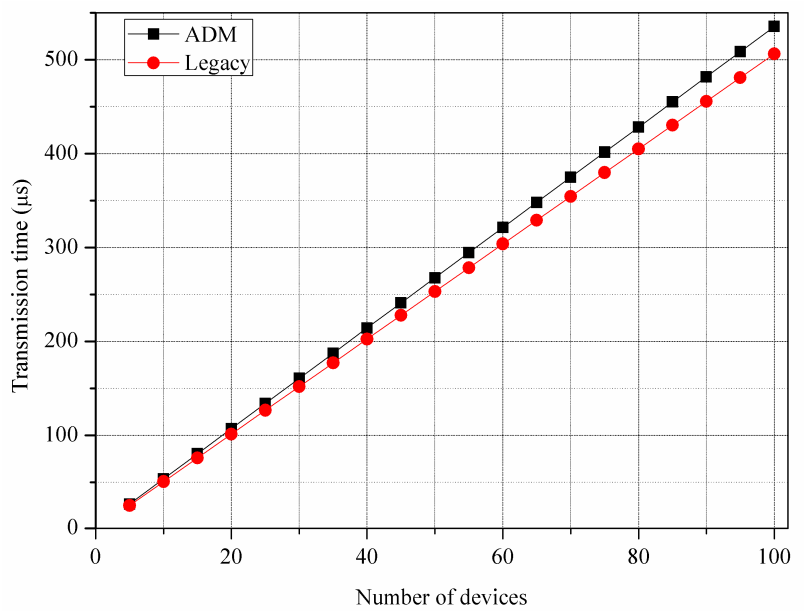

Figure 12. Transmission time for control message exchange. 
Table 4. Control overhead of device discovery.

\begin{tabular}{ccc}
\hline Control Overhead & ADM & Legacy \\
\hline Average energy consumption $(\mu \mathrm{W})$ & 117.01 & 115.98 \\
Average transmission time $(\mu \mathrm{s})$ & 11.59 & 11.49 \\
\hline
\end{tabular}

In Figures 13 and 14 to investigate the effect of the variation on the number of MGs, we consider the new simulation environments, where 100 devices are randomly deployed within $10 \times 10 \mathrm{~m}^{2}$ size room and they compose multiple MGs. The M2M GW transmits data packets of 4 Kbytes to each MG during the simulation time of $100 \mathrm{~ms}$.

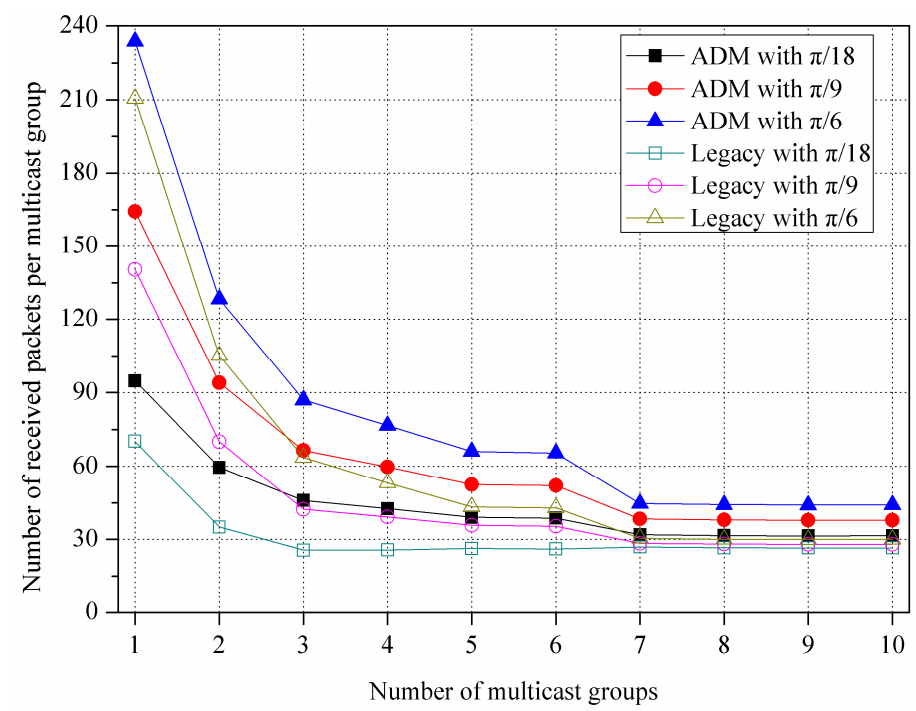

Figure 13. Number of received packets per multicast group.

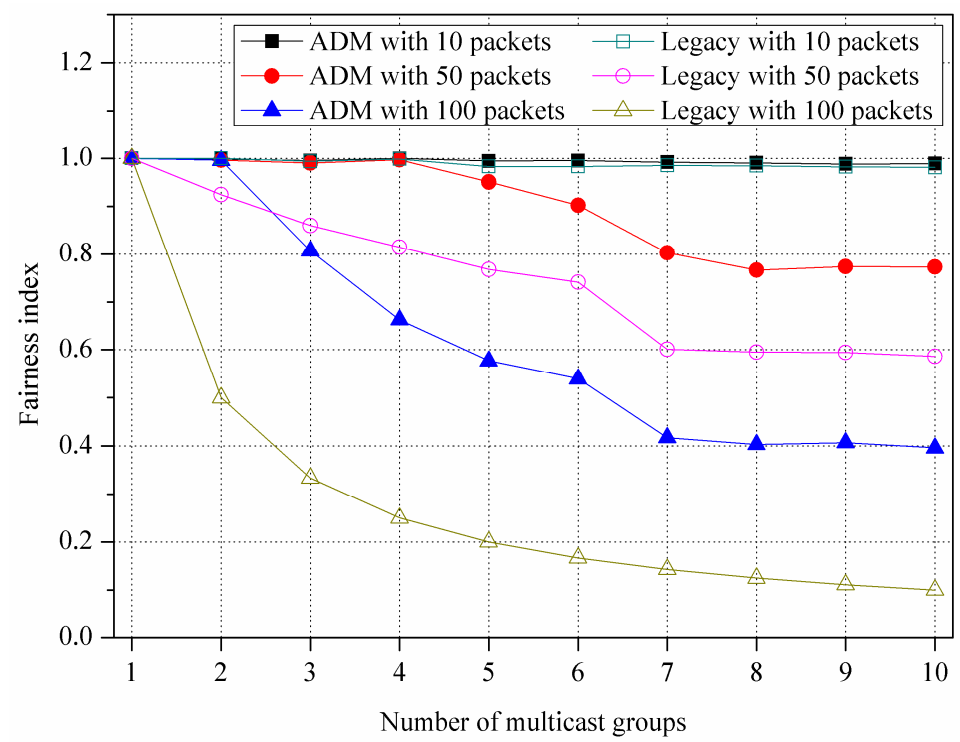

Figure 14. Variation of fairness index for various numbers of packets per multicast group.

Figure 13 shows the number of received packets per an MG for various unit-beam sectors. In this experiment, we assume that M2M GW transmits 10 packets to each MG sequentially. In both ADM and legacy multicast, the number of received packets per an MG decreases as the number of 
MGs increases. This is because a larger number of MGs are served from the M2M GW for the same simulation time (i.e., $100 \mathrm{~ms}$ ). In the figure, the devices running the ADM receive more packets than the legacy multicast because the ADM builds up a smaller number of transmission sectors by merging the adjacent unit-beam sectors via an asymmetric sectorization procedure, while the legacy multicast uses only the unit-beam sectors. Specifically, when the beamwidth of unit-beam sector is set to $\pi / 18$, $\pi / 9$, and $\pi / 6$, the ADM exhibits approximately $29.42 \%, 25.75 \%$, and $23.36 \%$ higher reception ratio of packets compared to the legacy multicast, respectively. Note that, in our simulated scenario, when the number of MGs is more than seven, it maintains constant. When the number of MGs increases, the average number of devices (i.e., MG members) per an MG is reduced, thereby also reducing the effect of the asymmetric sectorization. Thus, the number of received packets per an MG is no longer declining. Moreover, for the same reason, its difference between ADM and legacy multicast decreases as the number of MGs increases.

Figure 14 shows the fairness index for various numbers of packets per an MG. As mentioned earlier in Section 4.1, the number of packets per an MG means the number of chunks that are continuously transmitted in one transmission under HD video streaming application scenario, in which it is set to 10, 50, and 100, respectively. The fairness index $(F)$ can be calculated as follows [34]:

$$
F=\frac{\left(\sum_{i=1}^{n} x_{i}\right)^{2}}{n \sum_{i=1}^{n} x_{i}{ }^{2}}
$$

where $n$ is the number of MGs, $i$ is the MG ID, and $x_{i}$ is the fairness parameter, which means the number of packets that each MG receives. The fairness index of ADM starts to decrease at a larger number of MGs and keeps a higher value compared to that of legacy multicast, due to the benefit of asymmetric sectorization. Specifically, when the number of packets per an MG is 50 and 100, the ADM exhibits approximately $16.39 \%$ and $52.79 \%$ higher fairness index compared to that of the legacy multicast, respectively. Meanwhile, in the case that the number of packets per an MG is 10, both of the $\mathrm{ADM}$ and the legacy multicast can almost evenly serve all the MGs within the limited simulation time of $100 \mathrm{~ms}$, thus their fairness indices retain the value of one.

\section{Conclusions}

This paper presents asymmetric directional multicast transmission (ADM) for capillary M2M using mmWave communications. The optimum sectorization for a deployment pattern of multicast group members is achieved with ADM by merging the unit-beam sectors indicating the position of each M2M device. Thus, the minimum numbers of asymmetric sectors are built. The performance was evaluated by conducting experimental simulations with various factors that affect the network performance, such as variations in the beamwidth size of the unit-beam sector, the shape of room, and the position of M2M GW. The results of the simulation show that ADM can achieve considerable improvement in terms of the number of transmission sectors, transmission time, and aggregate throughput. In particular, ADM can be useful in real environments since it is shown to achieve a high performance in various room shapes and in different positions of M2M GW.

In our ongoing and future work, we plan to design the transmission scheduling strategy of ADM under the IEEE 802.15.3c MAC superframe environment and the optimization of the contention access period (CAP) duration for the system configuration will be discussed. Moreover, for future work, the theoretical analysis based on the mathematical modeling will be given to verify the effectiveness of this extended work and the accuracy of the simulations.

Acknowledgments: This research was supported by Basic Science Research Program through the National Research Foundation of Korea (NRF) funded by the Ministry of Education (NRF-2014R1A1A2057641). This research was also supported by Hallym University Research Fund, 2016 (HRF-201601-015). 
Author Contributions: Jung-Hyok Kwon and Eui-Jik Kim conceived and designed the protocol; Jung-Hyok Kwon implemented the protocol for the simulations and analyzed the simulation results; Jung-Hyok Kwon and Eui-Jik Kim wrote the paper; Eui-Jik Kim guided the research direction and supervised the entire research process.

Conflicts of Interest: The authors declare no conflict of interest.

\section{References}

1. Mehmood, Y.; Görg, C.; Muehleisen, M.; Timm-Giel, A. Mobile M2M communication architectures, upcoming challenges, applications, and future directions. Eurasip J. Wirel. Comm. 2015, 250, 1-37. [CrossRef]

2. Paul, A.; Rho, S.; Bharnitharan, K. Interactive scheduling for mobile multimedia service in M2M environment. Multimed. Tools Appl. 2014, 71, 235-246. [CrossRef]

3. Carretero, J.; García, J.D. The Internet of things: Connecting the world. Pers. Ubiquit. Comput. 2014, 18, 445-447. [CrossRef]

4. Kim, S.; Na, W. Safe data transmission architecture based on cloud for Internet of things. Wireless Pers. Commun. 2016, 86, 287-300. [CrossRef]

5. Kwon, J.-H.; Chang, H.S.; Shon, T.; Jung, J.-J.; Kim, E.-J. Neighbor stability-based VANET clustering for urban vehicular environments. J. Supercomput. 2016, 72, 161-176. [CrossRef]

6. Kim, T.-Y.; Kim, E.-J. Multi-hop WBAN configuration approach for wearable machine-to-machine systems. Multimed. Tools Appl. 2015. [CrossRef]

7. Huang, C.F.; Lin, W.C. Data collection for multiple mobile users in wireless sensor networks. J. Supercomput. 2015. [CrossRef]

8. Pandit, S.; Sarker, K.; Razzaque, M.A.; Sarkar, A.M.J. An energy-efficient multiconstrained QoS aware MAC protocol for body sensor networks. Multimed. Tools Appl. 2015, 74, 5353-5374. [CrossRef]

9. ISO/IEC/IEEE International Standard for Information Technology-Telecommunications and information exchange between systems-Local and metropolitan area networks-Specific requirements-Part 11: Wireless LAN Medium Access Control (MAC) and Physical Layer (PHY) Specifications Amendment 3: Enhancements for Very High Throughput in the $60 \mathrm{GHz}$ Band (adoption of IEEE Std 802.11ad-2012); IEEE: New York, NY, USA, 2012.

10. Standard ECMA-387 High Rate 60 GHz PHY, MAC and HDMI PAL. (2010). Available online: http:/ /www.ecma-international.org/publications/files/ECMA-ST/ECMA-387.pdf/ (accessed on 1 December 2015).

11. IEEE Std 802.15.3c-2009, IEEE Standard for Information technology-Telecommunications and information exchange between systems-Local and metropolitan area networks-Specific requirements. Part 15.3: Wireless Medium Access Control (MAC) and Physical Layer (PHY) Specifications for High Rate Wireless Personal Area Networks (WPANs) Amendment 2: Millimeter-wave based Alternative Physical Layer Extension; IEEE: New York, NY, USA, 2009.

12. Park, H.; Park, S.; Song, T.; Pack, S. An incremental multicast grouping scheme for mmWave networks with directional antennas. IEEE Commun. Lett. 2013, 17, 616-619. [CrossRef]

13. Schneider, T. Ultrahigh-bitrate wireless data communications via THz-links; possibilities and challenges. J. Infrared Millim. Te. 2015, 36, 159-179. [CrossRef]

14. Kim, W.; Song, T.; Pack, S. FRAS: Fair rate adaptation scheme for directional multicast in $60 \mathrm{GHz}$ multi-gigabit WLANs. Wireless Pers. Commun. 2014, 77, 1007-1017. [CrossRef]

15. Park, H.; Kang, C.-H. A group-aware multicast scheme in $60 \mathrm{GHz}$ WLANs. KSII T. Internet Info. 2011, 5, 1028-1048.

16. Wu, D.; Wang, J.; Cai, Y.; Guizani, M. Millimeter-wave multimedia communications: Challenges, methodology, and applications. IEEE Commun. Mag. 2015, 53, 232-238. [CrossRef]

17. Cai, L.X.; Cai, L.; Shen, X.; Mark, J.W. REX: A randomized exclusive region based scheduling scheme for mmWave WPANs with directional antenna. IEEE Trans. Wireless Commun. 2010, 9, 113-121. [CrossRef]

18. Park, H.; Park, S.; Shon, T.; Kim, E.-J. Multi-hop-based opportunistic concurrent directional transmission in $60 \mathrm{GHz}$ WPANs. Multimed. Tools Appl. 2015, 74, 1627-1644. [CrossRef]

19. Khatiwada, B.; Moh, S. A novel multi-channel MAC protocol for directional antennas in ad hoc networks. Wireless Pers. Commun. 2015, 80, 1095-1112. [CrossRef] 
20. Kwon, J.-H.; Kim, E.-J.; Kang, C.-H. CAD-MAC: Coverage adaptive directional medium access control for mmWave wireless personal area networks. In Proceedings of the 26th International Conference on Advanced Information Networking and Applications Workshops (WAINA), Fukuoka, Japan, 26-29 March 2012; pp. 751-754.

21. Park, H.; Kim, E.-J. Location-oriented multiplexing transmission for capillary machine-to-machine systems. Multimed. Tools Appl. 2015. [CrossRef]

22. Park, H.; Lee, C.; Lee, Y.S.; Kim, E.-J. Performance analysis for contention adaptation of M2M devices with directional antennas. J. Supercomput. 2015. [CrossRef]

23. Guo, S.; Guo, M.; Leung, V.; Yu, S.; Xiang, Y. On the multicast lifetime of WANETs with multibeam antennas: Formulation, algorithms, and analysis. IEEE Trans. Comput. 2014, 63, 1988-2001. [CrossRef]

24. Hou, Y.T.; Shi, Y.; Sherali, H.D.; Wieselthier, J.E. Multicast communications in ad hoc networks using directional antennas: A lifetime-centric approach. IEEE Trans. Veh. Technol. 2007, 56, 1333-1344. [CrossRef]

25. Zhang, G.; Xu, Y.; Wang, X.; Tian, X.; Liu, J.; Gan, X.; Yu, H.; Qian, L. Multicast capacity for VANETs with directional antenna and delay constraint. IEEE J. Sel. Areas Commun. 2012, 30, 818-833. [CrossRef]

26. Sen, S.; Ghosh, R.; Xiong, J.; Choudhury, R.R. BeamCast: Harnessing beamforming capabilities for link layer multicast. ACM SIGMOBILE Mob. Comput. Commun. Rev. 2009, 13, 34-37. [CrossRef]

27. Pyo, C.W.; Harada, H. Throughput analysis and improvement of hybrid multiple access in IEEE 802.15. 3c mm-wave WPAN. IEEE J. Sel. Area Comm. 2009, 27, 1414-1424.

28. Kim, J.; Mohaisen, A.; Hong, S.-N. Interference impacts on $60 \mathrm{GHz}$ real-time online video streaming in wireless smart tv platforms. Multimed. Tools Appl. 2015, 74, 8613-8629. [CrossRef]

29. Kim, T.; Kim, E.-J. View pattern-based adaptive streaming strategy for mobile content delivery services. Multimed. Tools Appl. 2015. [CrossRef]

30. Choi, M.; Lee, G.; Jin, S.; Koo, J.; Kim, B.; Choi, S. Link adaptation for high-quality uncompressed video streaming in $60 \mathrm{GHz}$ wireless networks. IEEE T. Multimedia 2013, 18, 627-642. [CrossRef]

31. Scott-Hayward, S.; Garcia-Palacios, E. High definition video in IEEE 802.15.3c mm-Wave wireless personal area networks. In Proceedings of the 36th International Conference on Local Computer Networks (LCN), Bonn, UK, 4-7 October 2011; pp. 93-100.

32. Singh, H.; Oh, J.; Kweon, C.; Qin, X.; Shao, H.R.; Ngo, C. A 60 GHz wireless network for enabling uncompressed video communication. IEEE Commun. Mag. 2008, 46, 71-78. [CrossRef]

33. Pandian, M.B.; Sichitiu, M.L.; Dai, H. Optimal resource allocation in random access cooperative cognitive radio networks. IEEE Trans. Mobile Comput. 2014, 14, 1245-1258. [CrossRef]

34. Babu, A.V.; Jacob, L. Fairness analysis of IEEE 802.11 multirate wireless LANs. IEEE T. Veh. Technol. 2007, 56, 3073-3088. [CrossRef]

(C) 2016 by the authors; licensee MDPI, Basel, Switzerland. This article is an open access article distributed under the terms and conditions of the Creative Commons Attribution (CC-BY) license (http://creativecommons.org/licenses/by/4.0/). 